\title{
The Case for Biological Quantum Computer Elements
}

\author{
Prof. Wolfgang Baer ${ }^{\mathrm{a}}$, Prof Rita Pizzi ${ }^{\mathrm{b}}$ \\ ${ }^{a}$ Dep. of Information Science Code IS, Naval Postgraduate School, 1 University Circle, Monterey, CA \\ 93943, Baer@nps.edu Tel 831-656-2209 \\ ${ }^{\mathrm{b}}$ Department of Information Technologies, University of Milan, \\ via Bramante 65, 26013 CREMA (CR) - ITALY \\ Tel. 390250330072 e-mail pizzi@dti.unimi.it
}

\begin{abstract}
An extension to vonNeumann's analysis of quantum theory suggests self-measurement is a fundamental process of Nature. By mapping the quantum computer to the brain architecture we will argue that the cognitive experience results from a measurement of a quantum memory maintained by biological entities. The insight provided by this mapping suggests quantum effects are not restricted to small atomic and nuclear phenomena but are an integral part of our own cognitive experience and further that the architecture of a quantum computer system parallels that of a conscious brain.

We will then review the suggestions for biological quantum elements in basic neural structures and address the de-coherence objection by arguing for a self- measurement event model of Nature. We will argue that to first order approximation the universe is composed of isolated self-measurement events which guaranties coherence. Controlled de-coherence is treated as the input/output interactions between quantum elements of a quantum computer and the quantum memory maintained by biological entities cognizant of the quantum calculation results.

Lastly we will present stem-cell based neuron experiments conducted by one of us with the aim of demonstrating the occurrence of quantum effects in living neural networks and discuss future research projects intended to reach this objective.
\end{abstract}

Keywords: Quantum Computers, Biological Quantum Effects, Whitehead, Process Ontology

\section{INTRODUCTION}

If a living brain can be shown to operate on quantum principles then it makes sense to examine its capabilities in order to learn how nature accomplishes such a feat and potentially extract biological components for the construction of quantum computers. This paper reviews several arguments addressing the possibility of quantum brain operation and describes an experimental approach for investigating this question. The case for quantum computation in the brain has been made through several lines of argument. These include the foundations-of-physics argument, the inadequacy of the neural switching model argument, and what I will call the process interpretation argument.

The foundations-of-physics argument is straight forward. Classic physics is an approximation to more accurate description of nature required to properly address phenomena at the atomic and molecular level. Since biology is based upon chemistry which in turn is based upon quantum physics, it is obvious that quantum theory must ultimately govern the activities in the human brain. As we delve deeper into the structure of the brain, components, whose operation can only be explained by quantum mechanical rules, must be present. Not surprisingly such components have been found and their investigation forms the main thrust of experimental research supporting the quantum mind theories proposed by Penrose ${ }^{[1]}$ and Hameroff. ${ }^{[2]}$ Discussions of ion transport mechanisms ${ }^{[3]}$ imply neural pulses are themselves subject to the uncertainties of quantum phenomena and strongly suggest that quantum effects plays a critical role in the formation and progress of our though patterns.

Unfortunately the discovery of components operating according to the rules of quantum theory does not prove the brain operates like a quantum computer any more than the presence of a tunneling diode turns a conventional computer into a quantum computer. All that such discoveries show is that lower level structures of nature can be encapsulated and harnessed into aggregates, which for all practical purposes operate on new emergent principles ${ }^{[4]}$. Research by several investigators ${ }^{[2]}$ have shown that components 
such as nano-tubules can perform quantum operations. However, attributing macroscopic brain functions, such as binocular rivalry ${ }^{[5]}$, to quantum operations is still highly speculative and cannot be taken as a strong argument, let alone as proof.

The second argument comes from the inadequacy of the neural switching model to explain brain operation. The difficulty encountered by neural scientist to explain cognitive brain functions has been addressed by many authors ${ }^{[6][7]}$. Despite much metaphoric success in the neural network arena, the neural pulse speeds and neuron counts are simply inadequate to account for the everyday brain operation. The performance of a basketball player or the image-recognition capacity of the human brain cannot be duplicated by computer operating near nano-second cycle times let alone the millisecond response times found in biological systems. For some, a more compelling argument is the inability of any classic physics based explanation to address the explanatory gap or what has been identified as the "hard problem of consciousness" ${ }^{[8]}$. Quite simply, the explanatory gap states that there is no explanation for the fact that you see these words in front of your nose. Photons entering your brain may produce a physical response in your neural structure, but how such a response then generates sensations several feet away cannot be explained within conventional physics. Compelling as these arguments may be, they only prove that brain operation is still a mystery. It is certainly attractive to connect the mystery of the brain with the mystery of quantum computation, but that is only a possibility waiting to be proved not a fact that has been proved by the argument.

\subsection{De-coherence and the Isolated System Model}

The argument against the possibility of quantum computation in the brain is based upon the inability to conceive of quantum computation in the warm soggy environment encountered in living tissue and is known as the de-coherence argument. Conceived in terms of conventional quantum theory the argument contends that a quantum state will collapse due to interaction with its environment. For biological systems the ambient coupling to a heat bath is roughly given by Boltzmann's constant times the absolute temperature of the systems. Since biological systems operate at room temperature the coherence length is roughly given by

$$
\Delta \mathrm{t}=\mathrm{h} / \mathrm{k} \cdot \mathrm{T}=6.6 \times 10-34(\mathrm{~J} \cdot \mathrm{sec}) / 1.38 \times 10-23 \mathrm{~J} / \mathrm{K} \cdot 300 \mathrm{~K}=1.6 \times 10-13 \mathrm{sec} .
$$

To perform useful quantum calculations logic gates must operate at time scales several orders of magnitude below this level. Alternatively quantum operations must be isolated in order to bring the effective temperature close to absolute zero or quantum error correction must be implemented to compensate for the untimely collapse.

Molecular isolation is being investigated by a number of investigators. The approach seeks to discover "protected" degrees of freedom within the neural molecular structure which could be considered isolated from its environment and therefore assigned an effective temperature near zero ${ }^{[9]}$. Unfortunately, though feasible in principle, actual sites at which the isolation might be happening in the neural environment has been difficult to identify let alone experimentally verify. In all cases the error rate is unacceptable for practical computation and hence a second avenue of approach is to look for quantum error correction schemes. The possibly is that unused segments of the DNA molecule may play a role as redundant components providing error correction. Unfortunately quantum error correction requires a large number of extra q-bits which in turn have errors and make the system more complex. Whether the additional reliability provided by error correction outweighs the complexity is an ongoing debate in conventional quantum computer circles where low temperature operation and isolation in reversible crystal structures are available. Conclusion by most physicists is that quantum computation is not feasible in the warm biological environment of the brain.

The situation is quite clear. If quantum theory, as currently conceived is the complete story, then the brain cannot operate like a quantum computer and its ability to generate thought and awareness remains a complete mystery for which no explanation exists even in principle. The key word in the above argument is the use of quantum theory as currently conceived by the Copenhagen School. Logically speaking, if our best theory of physics, cannot explain an obvious and every day phenomena then the assumption that such a theory is complete must be, as Einstein argued, incorrect and we must look for alternatives.

The third argument for quantum operation in the brain discussed in this paper is based upon a process interpretation of quantum theory that traces its origins to Whitehead's conjecture that fundamental 
events rather than fundamental particles are the building blocks of the Universe. This interpretation treats both the classic observer and the observed quantum system equally and therefore requires a reinterpretation of personal everyday experiences in quantum mechanical terms. We anticipate that this argument may be rejected outright by many workers because it introduces metaphysical and epistemological principles that do not conform to a strictly materialistic world view. It requires examining the properties of cognition and self awareness that have heretofore been excluded from physical inquiry. Before such rejection is finalized we would like to show that the very architecture of quantum theory suggests a process interpretation which, if proved correct, will greatly expand the applicability of physical theories to a larger range of observable phenomena than has been the case up to now.

\section{Process Interpretation}

The structure of quantum theory was initially analyzed by vonNeuman ${ }^{[10]}$ who defined a measuring Process I and a quantum Process II. Process II is governed by the Schrödinger Equation acting upon deBroglie waves and is deterministic in time. Process I is random and fraught with difficulties including the collapse of the wave function at the measurement instance, Schrödinger's Cat paradox, and the introduction of consciousness as the final measurement instrument. Active discussions of the measurement problem ${ }^{[11]}$ have lead to alternative, but by no means agreed upon interpretations, of quantum theory. Inspired by the writings of Henry Stapp ${ }^{[12]}$ I have extended vonNeuman's analysis by including a Process 0, corresponding to our classic world of action distributions governed by classic Hamilton's equations and a Process III originally suggested by Richard Feynman, by which the action distributions are converted into waves. This gives quantum theory a suggestive four processes architecture shown as a single connected activity in figure 1 below.

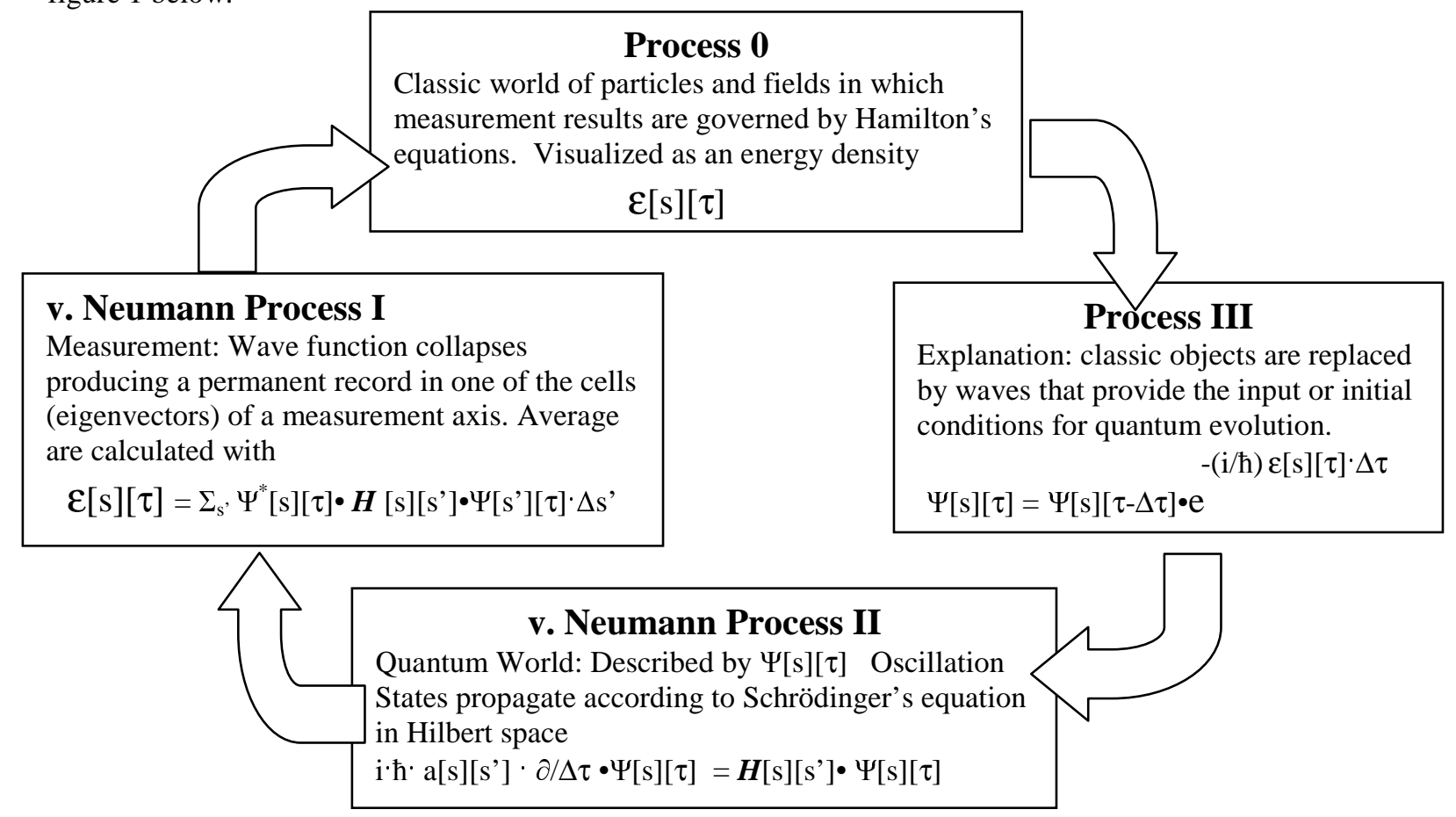

Fig. 1. - Process Architecture of Quantum Theory

Here each cycle represents the activity happening within a cycle of period $\Delta \tau$. The observable classic universe described by the action per cycle period, $\varepsilon[\mathrm{s}][\tau]$ is conventionally known as the energy density. The observations are explained by updating the deBroglie wave pattern $\Psi[s][\tau]$ which describe the quantum world through Process III. The energy in each part "s" is extracted by the measurement equation shown in Process I. An example of a two part energy operator is given by,

$$
\boldsymbol{H}[\mathrm{s}]\left[\mathrm{s}^{\prime}\right]=\left(\begin{array}{ll}
\mathrm{i} \cdot \hbar \cdot \mathrm{a}[0,0] \cdot \partial / \Delta \tau & \mathrm{i} \cdot \hbar \cdot \mathrm{a}[0,1] \cdot \partial / \Delta \tau \\
\mathrm{i} \cdot \hbar \cdot \mathrm{h}[1,0] \cdot \partial / \Delta \tau & \mathrm{i} \cdot \hbar \cdot \mathrm{a}[1,1] \cdot \partial / \Delta \tau
\end{array}\right)
$$


The off diagonal elements represent the interaction between parts. Hence Process I introduces interactions while Process III introduces time increment implemented by the process cycle. The functions, $\varepsilon[s][\tau]$ and $\Psi[s][\tau]$ represent two complementary ways to describe the universe. If one asks how $\Psi[s][\tau]$ propagates from cycle to cycle the answer is given by the Schrödinger equation shown in the Process II block of figure 1. If one asks how the energy density changes from cycle to cycle? The answer is,

$$
\mathrm{d} \varepsilon[\mathrm{s}][\tau] / \mathrm{d} \tau=(\partial \mathrm{H}(\mathrm{p}, \mathrm{q}) / \partial \mathrm{p})(\mathrm{dp} / \mathrm{d} \tau)+(\partial \mathrm{H}(\mathrm{p}, \mathrm{q}) / \partial \mathrm{q})(\mathrm{dq} / \mathrm{d} \tau)
$$

which equals zero, indicating no change in total action per cycle, if Hamilton's equations,

$$
\partial \mathrm{H}(\mathrm{p}, \mathrm{q}) / \partial \mathrm{q}=-\mathrm{dp} / \mathrm{d} \tau \quad \text { and } \quad \partial \mathrm{H}(\mathrm{p}, \mathrm{q}) / \partial \mathrm{p}=(\mathrm{dq} / \mathrm{d} \tau)
$$

are satisfied. As long as the cycle describes an isolated event the total action per cycle remains constant however the energy pattern as a function of space parts varies corresponding to the evolution of the classic universe. The quantum architecture shown in figure 1 replaces both the classic view of particles and fields as well as the traditional quantum view of deBroglie waves with and integrated self measurement cycle in which Nature continually displays the result of her measurement of her knowledge, $\Psi[\mathrm{s}][\tau]$, in the form of the classic universe described by $\varepsilon[s][\tau]$.

That such a process cycle can be mapped to the human consciousness traces its origins in the works of William James ${ }^{[13]}$. James identified conscious perception of everyday experience as a process. The

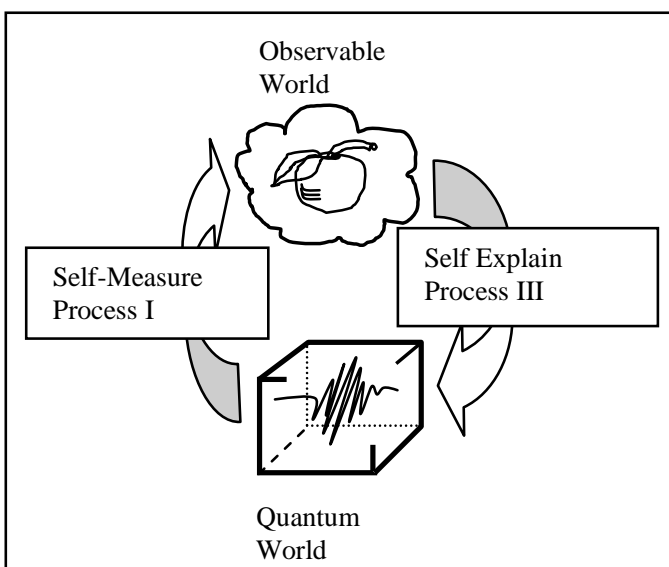

Fig. 2. Self-Measurement Model of the conscious process cycle conscious process was associated with a physical phenomenon by Alfred Whitehead ${ }^{[14]}$ in the early 20th Century. A more specific description of the conscious process is described ${ }^{[15]}$ under the heading of "Process Ontology", as a cycle of activity with mental experiences on one side and physical causes on the other. The connection between Process Ontology and quantum theory is discussed in many texts presenting Whitehead's theories ${ }^{[16][17]}$. This paper adopts Whitehead's idea of "actual entities" described by Philip Clayton as "Experiential units, constitutive of both process and objects, that are basic elements of reality" along with the notion that, "temporal change is a fundamental feature of the physical world itself", ${ }^{\text {"[18] }}$. A graphic representation of the fundamental selfmeasurement process shown in figure 2 maps directly into the architecture of quantum theory when vonNeumann's Process I is identified with self measurement, Process III is identified with self

explanation, and quantum world is taken for physical reality.

The similarity between the architecture of quantum theory and the psychological process of consciousness suggests an anthropomorphic knowledge system interpretation for quantum physics. This differs from the conventional hidden variable knowledge interpretation ${ }^{[19]}$ in that the classic universe of particles and fields is treated as a mental display while physical reality described in quantum theoretical terms is treated as a memory storage mechanism. The process interpretation suggests reality is built from the activity of becoming aware of ones knowledge and storing it into ones memory. What is diagrammed in figures 1 and 2 are the instructions executed by the "one" referred to in the last sentence and we will use the term Nature to refer to this executing system when necessary. The concept that Nature should be modeled as a self awareness activity has been popularized by many authors ${ }^{[20]}$. The development of useful physical theories based upon the process model ${ }^{[21]}$ and self-measurement ${ }^{[22]}$ notion is still a work in progress ${ }^{[23]}$. In the following section we will outline how the process interpretation can be related to the problem of quantum computer construction in qualitative terms. The presentation is intended to be suggestive and justify experimental investigations into the possibility of quantum effects in cognitive biological systems. 


\subsection{Mapping a Self-Measurement Activity to a Quantum Computer}

A quantum computer differs from a classic computer in that its memory bits consist of quantum systems or q-bits described by a wave function and its algorithms are implemented by quantum gates that apply unitary transformations in time. In the ion trap QC states of a Cs ion provide the q-bits which can be manipulated by laser pulses. Laser pulses provide controlled oscillating electromagnetic fields that induce transitions between Cs electron states. Atoms in different internal states interact with each other changing the transition energies between quantum levels. Each ions response to electromagnetic fields therefore depends upon the states of neighboring elements. This dependence allows quantum gates to be built from an array of ions. In our discussion and diagrams we will, for simplicity, show single ionized atoms ions, however systems of such components are actually being addressed.

The conventional quantum approach is to conceive of the "Observable World" in terms of a classic laboratory described by classic particles and fields and the "Quantum World" as a displacement field of deBroglie waves. However this approach mixes metaphors and leads to inconsistencies and paradoxes, such as Schrödinger's Cat Paradox, which we will attempt to correct with our process approach. Accordingly the whole is self-measurement cycle and we treat an external world as a division The single self-measurement cycle shown in figure 2 can apply only to completely isolated systems, such as the universe as whole, or idealized approximations of isolated sub-systems. For our discussion we divide the universe into two cycles labeled Atom and Nature. A diagram of a two part division is shown in figure 3. Since the whole must be equal to the sum of its parts plus all their interactions we get two independent selfmeasurement cycles connected by two interaction activities. Here the energy pattern, $\varepsilon[\mathrm{N}]$, seen by Nature is depicted by atomic orbitals while its knowledge of the atom, $\Psi[N]$, is depicted by a deBroglie wave in Hilbert Space. Similarly the energy pattern seen by the atom, $\varepsilon[\mathrm{A}]$, is depicted by icons of charged particles +/- and small stars depicting gravitational fields.

The important insight is that the classic and conventional concept of the atom as a system of particles has been relegated to Natures display space in which an energy pattern, $\varepsilon[\mathrm{N}]$, may be recognized as a particle. This recognition is clearly part of the Nature's accommodation to the other self-measurement cycles, outside of its own awareness, not to the independent existence of objects. This is a point Niels Bohr made consistently in his discussions about quantum theory. The atom, as a classic object, does not exist independently but is brought into existence by the act of measurement. In our interpretation we clearly see the classic atom, conceived as an object, is part of Natures knowledge i.e. an energy pattern held in its own cycle of activity. The "real" atom is another cycle of activity happening completely outside the boundaries of Nature as defined above.

To more closely relate our graphic drawings with the mathematics of quantum theory let us consider an interaction between these two event cycles. Initially both the atom and nature are in stationary states. In this case there is no interaction in Process I and the action per cycle in both the atom and Nature will remain constant and can be calculated from the residual knowledge each system has of the other.

$$
\begin{aligned}
& \varepsilon[\mathrm{N}]=\left[\Psi[\mathrm{N}]^{*}, \Psi[\mathrm{A}]^{*}\right]\left[\begin{array}{cc}
\boldsymbol{H}[\mathrm{N}][\mathrm{N}] & 0 \\
0 & \boldsymbol{H}[\mathrm{A}][\mathrm{A}]
\end{array}\right]\left[\begin{array}{l}
\Psi[\mathrm{N}] \\
\Psi[\mathrm{A}]
\end{array}\right]
\end{aligned}
$$


Assume that an interaction is turned on for one cycle by, for example firing a laser pulse into the location of its knowledge of the atom. Then interaction energy will introduce off diagonal terms in the energy matrix and the resulting actions per cycle will change as follows.

$$
\begin{gathered}
\varepsilon[\mathrm{N}]=\varepsilon[\mathrm{N}, \mathrm{N}]+\varepsilon[\mathrm{N}, \mathrm{A}]=\Psi[\mathrm{N}]^{*} \cdot \boldsymbol{H}[\mathrm{N}][\mathrm{N}] \cdot \Psi[\mathrm{N}]+\Psi[\mathrm{N}] * \boldsymbol{H}[\mathrm{N}][\mathrm{A}] \cdot \Psi[\mathrm{A}] \\
\varepsilon[\mathrm{A}]=\varepsilon[\mathrm{AA}]+\varepsilon[\mathrm{A}, \mathrm{N}]=\Psi[\mathrm{A}]^{*} \cdot \boldsymbol{H}[\mathrm{A}][\mathrm{A}] \cdot \Psi[\mathrm{A}]+\Psi[\mathrm{A}]^{*} \cdot \boldsymbol{H}[\mathrm{A}][\mathrm{N}] \cdot \Psi[\mathrm{N}] \\
\\
\Psi[\mathrm{N}][\tau]=\Psi[\mathrm{N} / \mathrm{h})(\varepsilon[\mathrm{N}, \Delta \tau] \cdot \mathrm{N}][\tau]+\varepsilon[\mathrm{N}, \mathrm{A}][\tau]) \Delta \tau
\end{gathered}
$$

If during the next cycle the interaction is turned off the diagonal terms in Process III drop out again and by applying the definition of the energy operator to the wave form of equation (7) we can extract a constant new energy level as shown below.

$$
\varepsilon[\mathrm{N}, \mathrm{N}][\tau-\Delta \tau]=\varepsilon[\mathrm{N}, \mathrm{N}][\tau]+\varepsilon[\mathrm{N}, \mathrm{A}][\tau]=\Psi[\mathrm{N}][\tau] \cdot \mathrm{i} \cdot \hbar \cdot \mathrm{a}[0,0] \cdot \partial / \Delta \tau \cdot \Psi[\mathrm{N}][\tau]
$$

A similar equation applies to the atom so after an interaction both systems execute stationary cycles at new energies and new deBroglie wave displacement states. Since we have only used a

\begin{tabular}{|c|c|c|c|c|}
\hline $\mathrm{H}[\mathrm{N} 1, \mathrm{~N} 1]$ & $\mathrm{H}[\mathrm{N} 1, \mathrm{~N} 2]$ & $\mathrm{H}[\mathrm{N} 1, \mathrm{~N} 3]$ & 0 & 0 \\
\hline $\mathrm{H}[\mathrm{N} 2, \mathrm{~N} 1]$ & $\mathrm{H}[\mathrm{N} 2, \mathrm{~N} 2]$ & $\mathrm{H}[\mathrm{N} 2, \mathrm{~N} 3]$ & 0 & 0 \\
\hline $\mathrm{H}[\mathrm{N} 3, \mathrm{~N} 1]$ & $\mathrm{H}[\mathrm{N} 3, \mathrm{~N} 2]$ & $\mathrm{H}[\mathrm{N} 3, \mathrm{~N} 3]$ & 0 & 0 \\
\hline 0 & 0 & 0 & $\mathrm{H}[\mathrm{A} 1, \mathrm{~A} 1]$ & $\mathrm{H}[\mathrm{A} 1, \mathrm{~A} 2]$ \\
\hline 0 & 0 & 0 & $\mathrm{H}[\mathrm{A} 1, \mathrm{~A} 1]$ & $\mathrm{H}[\mathrm{A} 1, \mathrm{~A} 2]$ \\
\hline
\end{tabular}
two part division the gain of action in one system must be exactly balanced by the loss in the other since the total amount of action in the whole universal event must be constant since it is assumed to be isolated by

Fig. 4- Two Universe Divisions with Interacting subsystems definition of the term 'whole'. If further divisions are introduced the action can transfer through sequential stages as a non symmetric energy flow. Figure 4 shows an example of Nature and the Atom though not interacting with each other contain internal interacting parts. Specifically a two state Atomic system can be used as a Q-bit while an array of such systems, formally introduced by adding $2 \times 2$ matrices along the diagonal of figure 4 , can act as a register or memory of a quantum computer. Interactions between such two state systems can further split the energy states and thus provide quantum gates required for logical operation implementation and produce entangled states required for quantum parallelism.

\subsection{Visualization of Quantum Computation in the Process Model}

The change from conventional quantum theory implied by figure 4 is that no vonNeumann cut is needed and no classic system is required to actualize the probabilities describing the quantum segment.

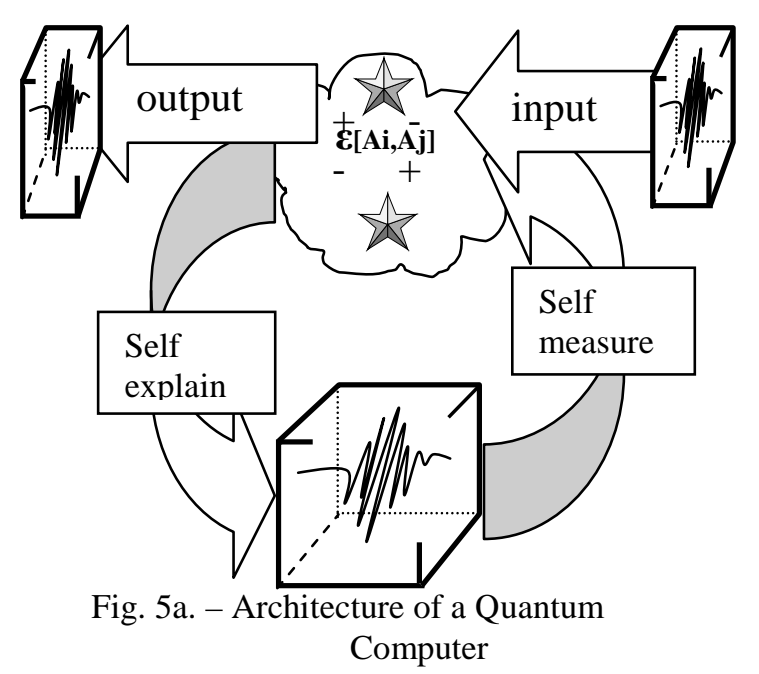
Instead each of the two parts contains a self measurement mechanism which actualizes itself. In figure 3 each of the cycles were labeled with single space names " $\mathrm{N}$ " and "A" and treated as single systems with single values for $\varepsilon[\mathrm{N}]$ and $\varepsilon[\mathrm{A}]$. The energy measurement result from a single system should have been graphically represented by a single color in their respective display spaces. Only after sub-divisions suggested by figure 4 introduces parallel cycles labeled "Ni" and "Ai" can the content of the measurement display spaces contain discernable details. With subdivisions providing a space of parallel cycles the display space can correctly contain a picture of a measurement result as complex as the result of any calculation a systems can perform through physical evolution. For a quantum computer 
this physical evolution is described as a unitary operator implementing in time by sequential applications of the self measurement cycle. In this visualization a quantum computer is a cycle sequence in which the value of the i'th memory cell during each cycle is simply the sum of all its internal interactions

$$
\varepsilon[\mathrm{Ai}]=\sum_{\mathrm{Aj}} \varepsilon[\mathrm{Ai}, \mathrm{Aj}]
$$

and the progress of the calculation is equated with the evolution of the energy pattern as a function of the internal cycle counter called time. The same status is accorded to the cycle the rest of the universe, we have called Nature, executes. By truncating the interaction branches in figure 3 we get a diagram shown in figure 5 a that represents a quantum computer in a more conventional form. The interaction with Nature is here seen as the input and output branches while the actual calculation is performed during the execution of the atomic-system clock cycles. It should be pointed out that the input and output are separated in time not necessarily space and when this time interval is measured in Natures clock the number of seconds required to perform a calculation internally to the A-system is not well defined. If the $\varepsilon$ [Ai] pattern does not change from cycle to cycle the system is stationary and time as measured by an internal clock does not advance.

In fact since you the reader are presumably part of Nature nothing that happens in the A-system is directly known and it is instructive to describe the quantum computer operation as seen from Nature's point of view. For simplicity we will again assume the external quantum computer is an atomic system shown as Bohr atom icon in Natures display space in figure 5b. It is mathematically described by its action per cycle density $\boldsymbol{\varepsilon}[\mathbf{s}, \mathbf{t}]$ where "s" labels space divisions in Nature and " $\mathrm{t}$ " names its states and/or the cycle in which such a state is stable. This energy pattern is equal to the orbital density calculations made for atoms and molecules. The explanation of this display space appearance is a displacement pattern $\Psi[\mathbf{s}, \mathbf{t}]$ in Natures Hilbert space which is calculated from Natures experience by the explanation Process III. The space and time labels here are the same which reflects the fact that action at a space time point labels a cycle and connects a measurement result display space point with its Hilbert space cell counterpart in the process model.

Assuming Natures Hilbert space points can be modeled as small systems each named "s" and the motion in one cell is coupled to neighboring cells by interaction potentials, then for small enough interactions the resulting coupled disturbance inside each cell can be described by a wave function $\Psi[\mathrm{s}, \mathrm{t}]$ and equations governing the resulting coupled disturbance is a Schrödinger equation in the non-relativistic approximation. The last statement is the basis of Schrödinger's original derivation and its classic connection can best be understood by reading Goldstein's excellent Classic Mechanics text chapter 10 on Small Oscillations. By adjusting the inter-space-cell potentials Nature can force the wave patterns into stable activities which hold its knowledge of the A-system. By measuring the action content of this knowledge through Process I Nature will experience an energy density corresponding to the A-systems objective visualization.

Here again the important insight: The A-system as experienced by Nature and by analogy the visualization of the A-system experienced by the reader when taking on the role of Nature is not an external independent entity but rather Nature's internal accommodation whatever is the actual external entity. That actual entity has been postulated, in the process model, to be the cycle shown completely outside of Nature in figure 5 a. Nature maintains knowledge of this external entity in its own space cycles and visualizes that knowledge as energy paterns but these internal activities are Nature's adjustment to the existence of an outside entity not the entity itself.

Once it is clear that the energy pattern represented by a p-electron orbital icon in figure $5 \mathrm{~b}$ is not external to Nature it is safe to identify this experience in Natures display space as the 'classic observable' and Natures display space as "classic inertial space", both of which are correctly treated within the limits of classic physics. Within these limits Nature is presumed to

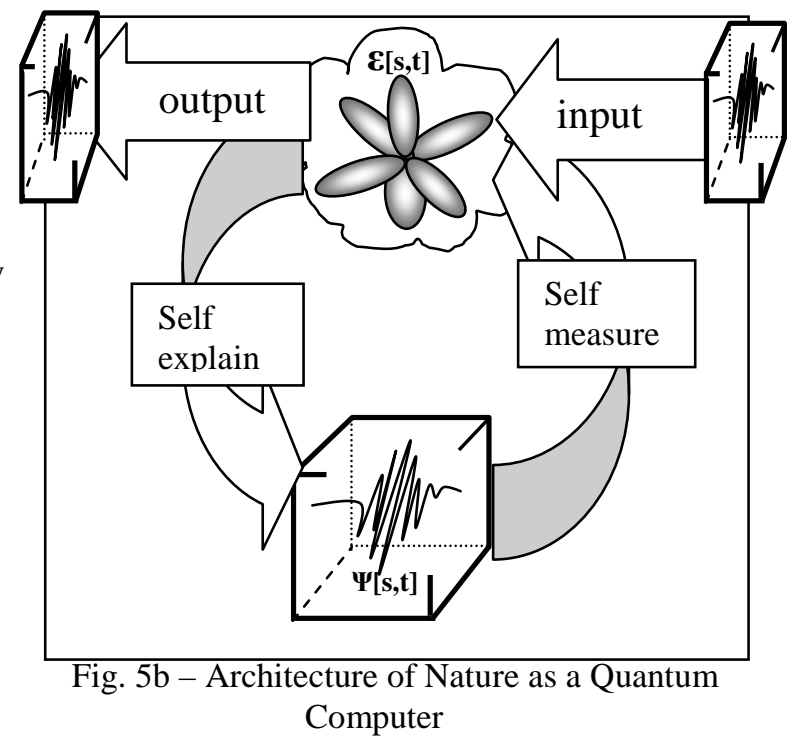
have complete and accurate knowledge and the very idea that an additional external cycle is required seems 
superfluous. However once we recognize that, what we had though was a "classic observable", is actually Natures knowledge display and what was a deBroglie wave is actually Natures way of storing its knowledge, then it also becomes obvious that uncertainty can lead to entanglement, the collapse of the wave function, and quantum parallelism.

The interpretation of what Nature does when it acts like a computer is to calculate what it expects to measure if it where to interact with the external system i.e. make a real measurement. It performs this calculation by making an internal measurement of its knowledge of such an external system. If it does not know the exact state of the external system then the best it can calculate is alternatives. This leads to energy patterns smeared out between alternative possibilities. These energy patterns are real. Nature's expectation of where a particle should interact is actually where it will show up unless Nature is uncertain of the particle state. Remember particle here is used not to refer to Natures expectation or its knowledge but in the classic sense of the external cause i.e. the external cycle. It is the state of this cycle that may not be known inside Nature. In this case all Nature can do is to calculate all possibilities. Then when the particle shows up in one of the calculated possibilities Nature updates its knowledge accordingly by absorbing a quantum of change.

Thus when building a quantum computer we are manipulating the properties of entities we had been thinking where classic observable by treating their causes as quantum mechanical systems. In the process model we identify these two items as Natures expectations of measurement interactions and Natures knowledge of external systems. When we query Nature - or more accurately we query measuring instruments built from rearrangements of Nature - we get what Nature knows of external systems. In more classic terminology physicists do not look at things themselves but rather at the results of recordings in measurement coordinate frames. These coordinate frames are parts of Nature and what we extract from them are Natures knowledge. If correct, the process model suggests that the whole should be treated as an event and divided into self measuring events which are known to each other only through interpretations and theories of interactions held inside themselves as knowledge or perhaps more accurately as best guesses. The parts of a process universe are more like the first, second, third, etc. persons acting like interacting quantum computers, while classic objects are knowledge items in these persons. In the process model the A-System discussed above is an independent activity cycle outside of Nature that has an objective representation inside of Nature and some primitive properties of self awareness derived from the architecture of its process activities. Therefore in the process model a more sophisticated cognitive system like a human being would also be constructed from an independent activity cycle and interact with Nature to produce an objective representation inside of Nature. This objective representation would show up as objective observables and material structures that would be recognized as the brain. If the brain is Natures knowledge representation of an external cognitive cycle it, like the A-system above, would operate like a quantum computer and Nature would have solved the difficult construction and de-coherence problems in a warm soggy environment and a search for biological quantum computing elements makes sense. Such elements could be used either to enhance our understanding and guide our construction of quantum computing elements or be used directly as building blocks in such systems. The following section discusses several experiments conducted by one of us to explore the possibility of quantum operation in biological systems.

\section{EXPERIMENTAL APPROACH TO QUANTUM BRAIN PHENOMENA}

Experimental proof that the brain is a quantum computer requires the discovery of brain phenomena that can only be explained by evoking quantum models. We classify approaches as internal or external.

Internal approaches are psychological in nature and are characterized by the utilization of the input and output circuitry naturally available in human beings. Experiments typically provide setup instructions, stimulus, and response measurements to a cooperating subject. The idea is to develop models of processes that must be going on in the brain that explain the response from a subject given a controlled set of input. The vast majority of investigations along these lines have sought to fit neural circuitry models to response data thus assuming the brain acts like a classic computer. The inadequacy of this assumption was discussed in the introduction. It is doubtful that our ability to perform pattern recognition can be implemented with pulses traveling at $100 \mathrm{~m} / \mathrm{sec}$ and even more doubtful that our ability to generate representations of objects at far distances from our brains can be explained in classic terms. 
The use of quantum models to explain brain response is being addressed by a growing number of investigators. Investigation of the brain as a quantum-like computer ${ }^{[7]}$ avoids the need for exact quantum mechanical representations of large bulk phenomena by treating the brain as quantum-like and appealing to metaphoric similarity. This is essentially the argument we developed in the last section. A recent paper ${ }^{[24]}$ has gone a step farther by calculating the probability distribution of dominance duration of rival states in binocular rivalry from known values of neuronal oscillation frequencies and firing rates using orthodox quantum theory. In binocular rivalry the brain is stimulated with simultaneous but different left and right eye scene input. The three dimensional scene generated by the brain will switch between rationalizing the left and right input with a characteristic frequency. The frequency was calculated by assuming the left and right interpretation corresponds to orthogonal quantum brain states that transform into each other according to quantum mechanical rules.

The internal psychological approach sends signals into the system of investigation and draws its conclusions from what comes out. This leaves the complex layer of classic neural pathways between the external stimulus and the presumed gateway to the quantum domain. Since the exact operation of this preprocessing layer is not fully understood, it is very difficult to draw conclusions about quantum operations. Some, perhaps undiscovered, algorithm executed by classic switching circuit could explain most evidence. This difficulty can be overcome by directly measuring neuron signals at the source. Since 2002, a group headed by one of us ${ }^{[25][26][27]}$ has been concerned with the direct acquisition of signals from cultured neurons in Micro-Electrode Arrays (MEAs). MEAs allow neurons to grow directly on electrodes imbedded in petri dishes. The signals of living cells can then be monitored. During the first experiments, we noticed anomalies in the electrical signals coming from separate and isolated neural cultures that suggested that either neurons were extremely sensitive to classical electromagnetic stimulation or some form of entangled state communication between isolated systems was occurring.

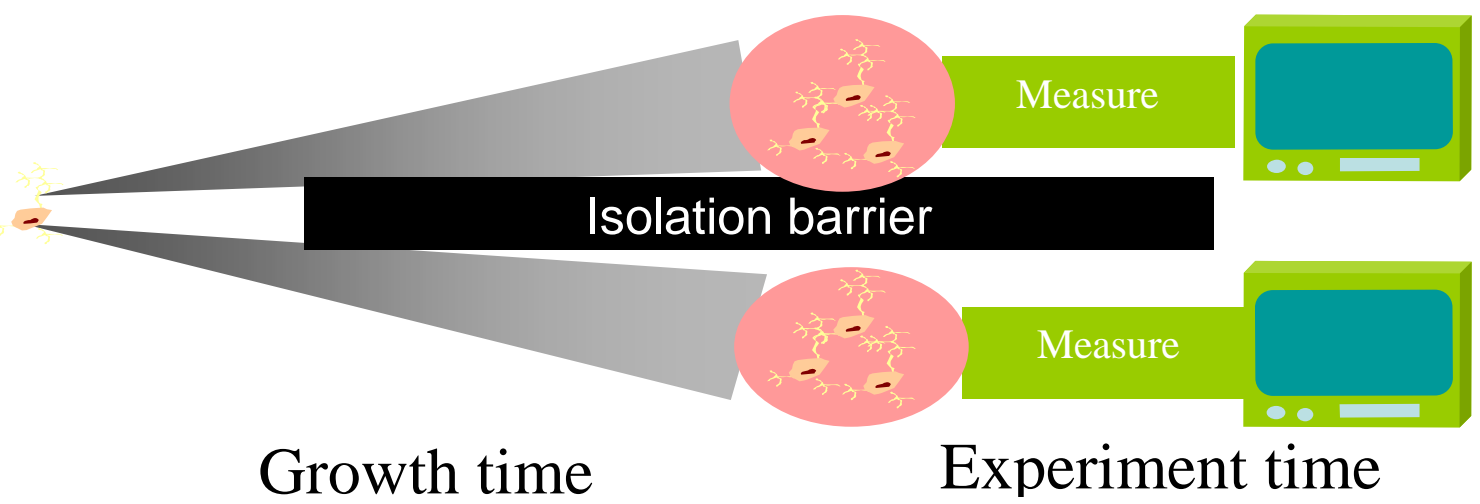

Fig. 6- Correlated signals from entangled states in living cells

The case for entangled state communication is highly speculative but can be understood as follows. Figure 6 shows a diagram of two neural cultures starting on the left with an initial single stem cell. As the culture grows identical systems are manufactured, which presumably interact, producing complex entangled states. The neurons, or sub-elements such as the DNA or microtubules, form the Hilbert space of a quantum system. At some time during the growth process the cultures are separated however, entangled state communication between them could remain.

Entangled state communication can be understood as signals travelling backward ${ }^{[28]}$ in time through the common origin of interaction. For such communication paths to remain intact some portion of the systems would have to remain isolated for extended periods of time. The classic world view would eliminate this possibility because of thermal interaction between the neurons and the rest of the universe. However if our Quantum Universe conjecture is correct all forms but specifically living potentially conscious entities are, to first approximation, isolated systems and only their interaction pathway extensions are accessible and subject to thermal interaction and de-coherence. Hence if the two neuron cultures could be completely isolated from each other and signals measured that show a high degree of correlation, the possibility of long term isolation and quantum based communication would be indicated. This is the hypothesis that such an experiment is designed to investigate. 
The basic experimental setup for the non-local experiment is shown in Figure 7. Two Microelectrode Array Basins containing living neurons grown from a single stem cell are separated by a distance $\mathrm{D}(8 \mathrm{~cm})$. An electromagnetic shield is placed around one basin and data acquisition cables, so no detectable leakage, crosstalk or induction between the basins occur. Voltages of independent neural activity

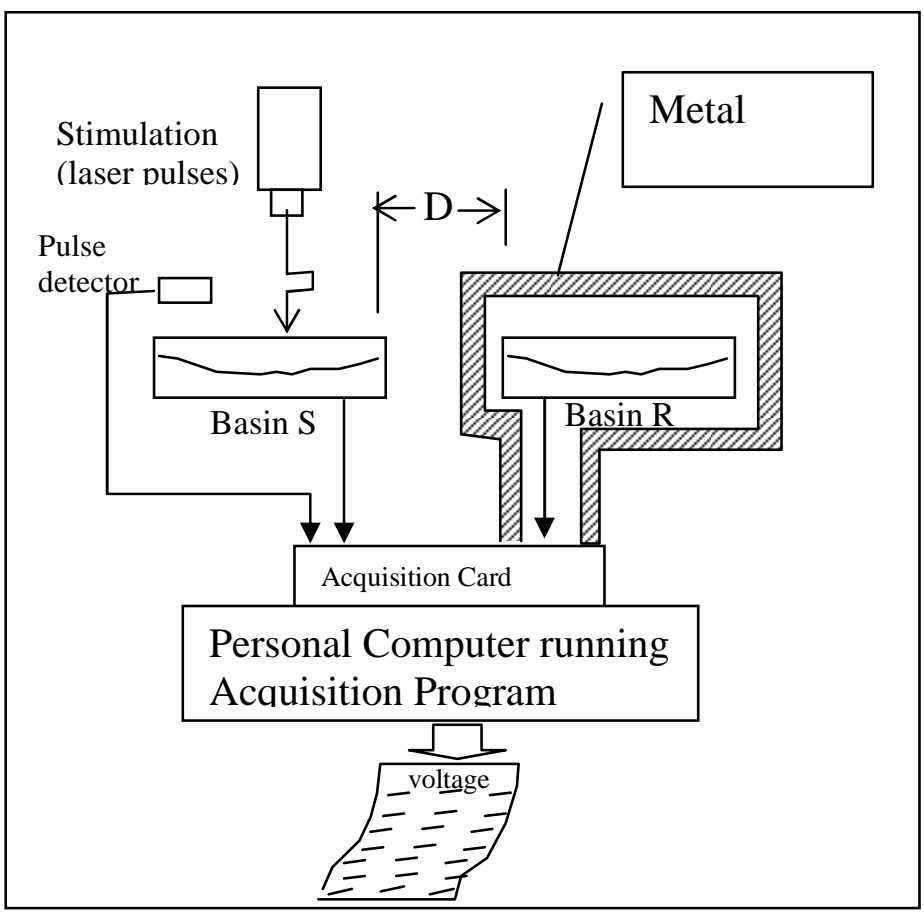

Fig. 7. Experimental Setup are measured and recorded for each basin. Stimulation of basin-S using an 80 milli-second laser pulse is monitored by Pulse detector. Voltages from the pulse detector, basin-S, and basin-R are sampled $(1 \mathrm{khz})$ and recorded in computer files. The files are then analyzed for signal patterns indicating communication has occurred between the two basins. The result of this analysis claims communication not attributable to electromagnetic origins does occur. We are not talking difficult statistical analysis or subtle occasional coincidences. This claim is based upon easily observed and obvious signal responses in the receiver basin-R.

Figure 8 shows a plot of the first 30 seconds of voltages taken at $1 \mathrm{khz}$ intervals. Plots for the pulse stimulated basin-S and receiver basin- $R$ are shown. Random laser pulses occur near 1809, 13561,14465,23505,25313, and 26217 milliseconds from $30000 \mathrm{~ms}$ start-time shown on the dark upper line. The response notch from the stimulated basin shown on the magenta second line and the receiver basin shown on the yellow third line can be made out. We can expand the graphs around the pulse location in order to see the wave forms more clearly. This was done in figure 9.

To see the amplitude response more clearly we run the data through a low pass filter averaging 40 samples at a time. The plot of the running average is shown in figure 10 . The $80 \mathrm{~ms}$ laser pulse is converted to a diagonal drop, flat bottom, and diagonal rise. The magenta middle line shows the stimulated Basin-S responding with a delay. The bottom yellow line shows the receiver Basin-R responding with a corresponding delay.

\section{Fig. 8 October Experiment Summary 30000 to $60000 \mathrm{msec}$}

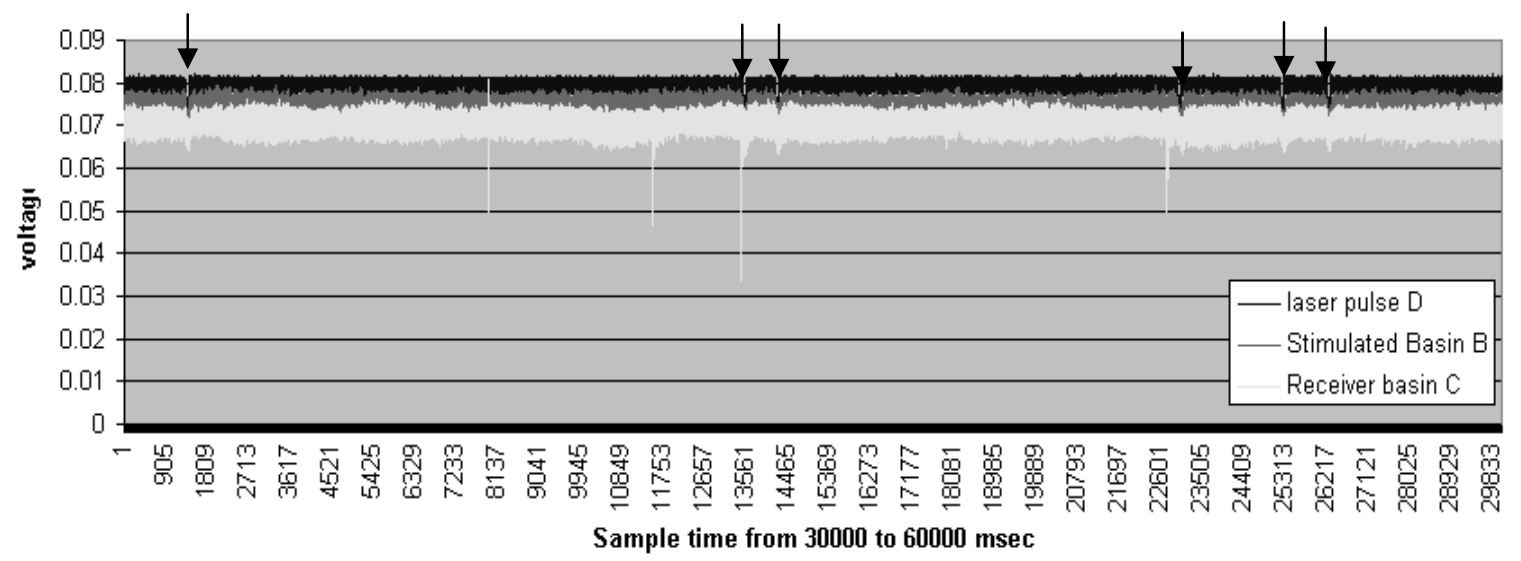




\section{Fig. 9 October Experiment 400 ms Pulse Expansion}

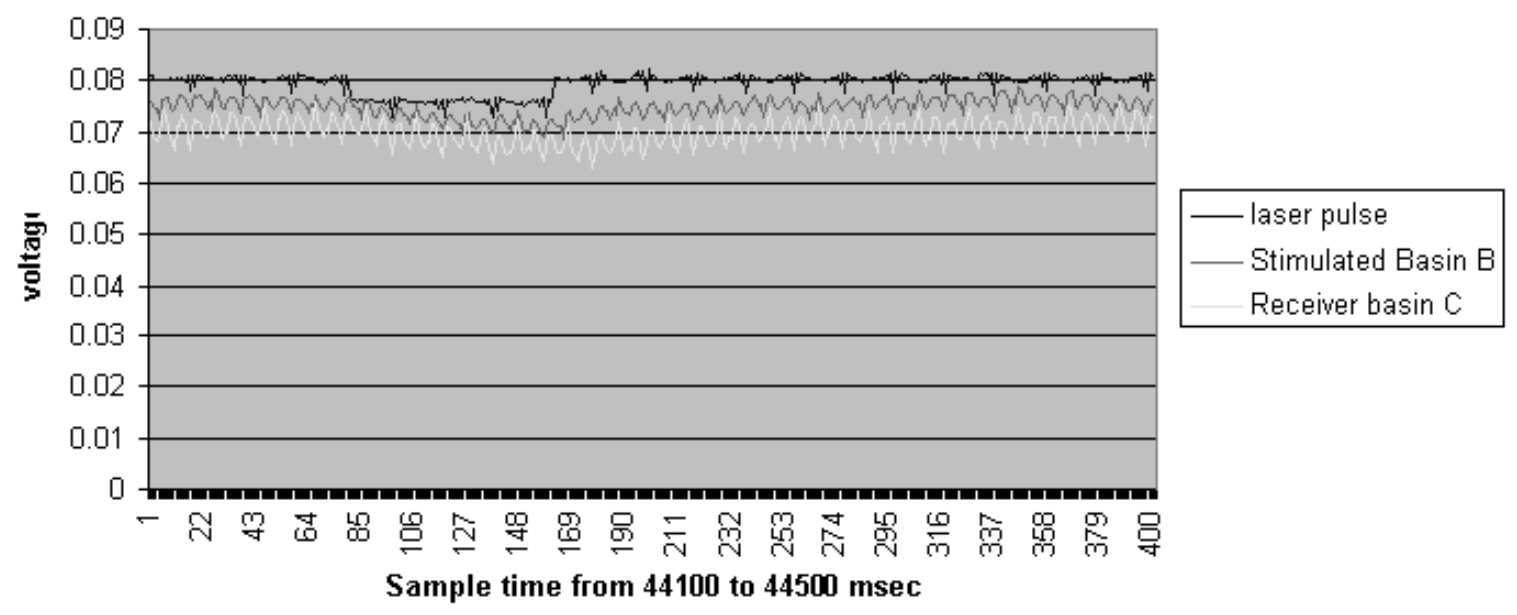

Fig. 10 October Experiment $400 \mathrm{~ms}$ Running 40ms Ave. Pulse Expansion

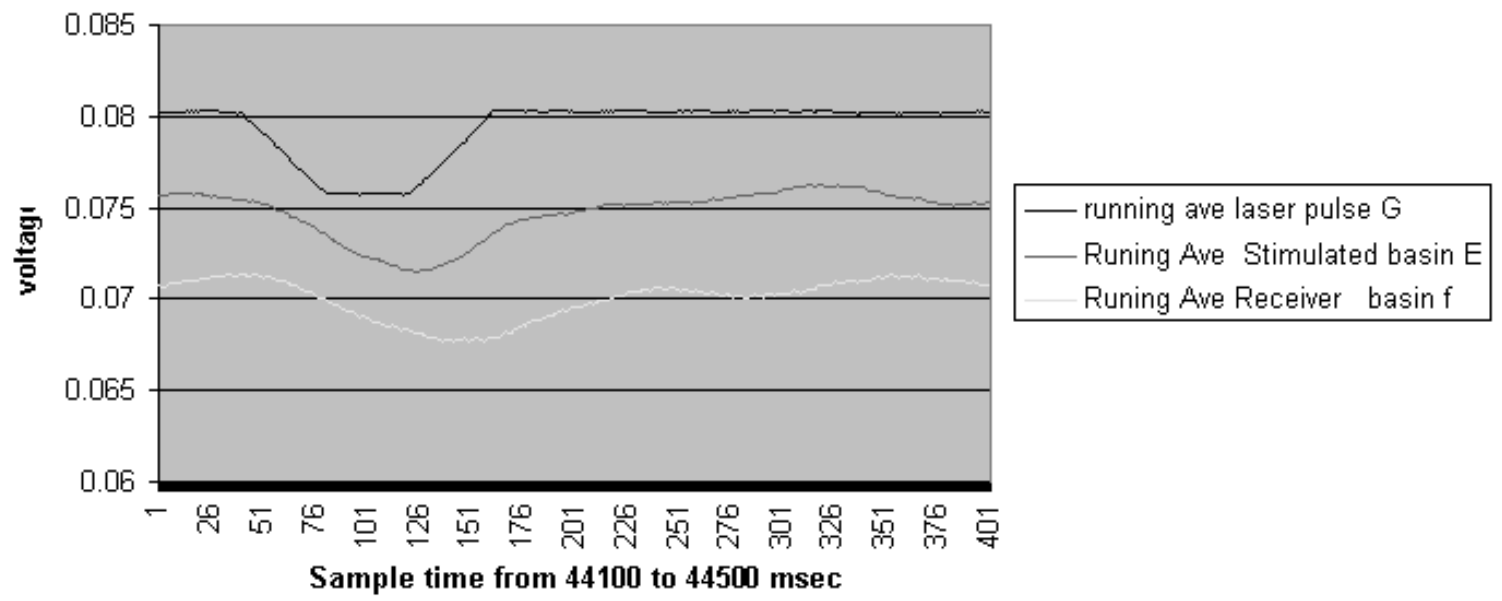

The graphs shown above provide visual examples of individual laser stimulation pulses along with the response in the stimulated and isolated receiver basin. Although visual inspection clearly shows wave form correlations a simple statistics performed on ten pulses in the first minute of data show a consistent voltage drop and delay as the stimulation works its way through first Basin-S and subsequently to Basin-R.

Table 1 Voltage and Delay for 10 pulses

$\begin{array}{lcc} & \text { AVE } & \text { STDEV } \\ \text { Delay between Pulse center and Stimulated basin center } & 24.7 \mathrm{msec} & 2.2 \mathrm{~ms} \\ \text { Delay between Stimulated and Received basin center 16.7msec } & 9.6 \mathrm{~ms} & \\ \text { 100ms average Laser Pulse Voltage drop } & 3.1 \mathrm{mv} & .5 \mathrm{mv} \\ 100 \mathrm{~ms} \text { average Stimulated Voltage drop } & 2.8 \mathrm{mv} & 1.0 \mathrm{mv} \\ 100 \mathrm{~ms} \text { average Receiver Voltage drop } & 1.5 \mathrm{mv} & 1.0 \mathrm{mv}\end{array}$

Though many additional checks and tests could be done, we believe the data showing $1 \mathrm{~ms}$ samples from laser, stimulated and received locations show clear evidence of correlation between the signals. The next question to be asked is whether the experiment was conducted with sufficient care to justify the conclusion that non-electromagnetic communication has been observed. 


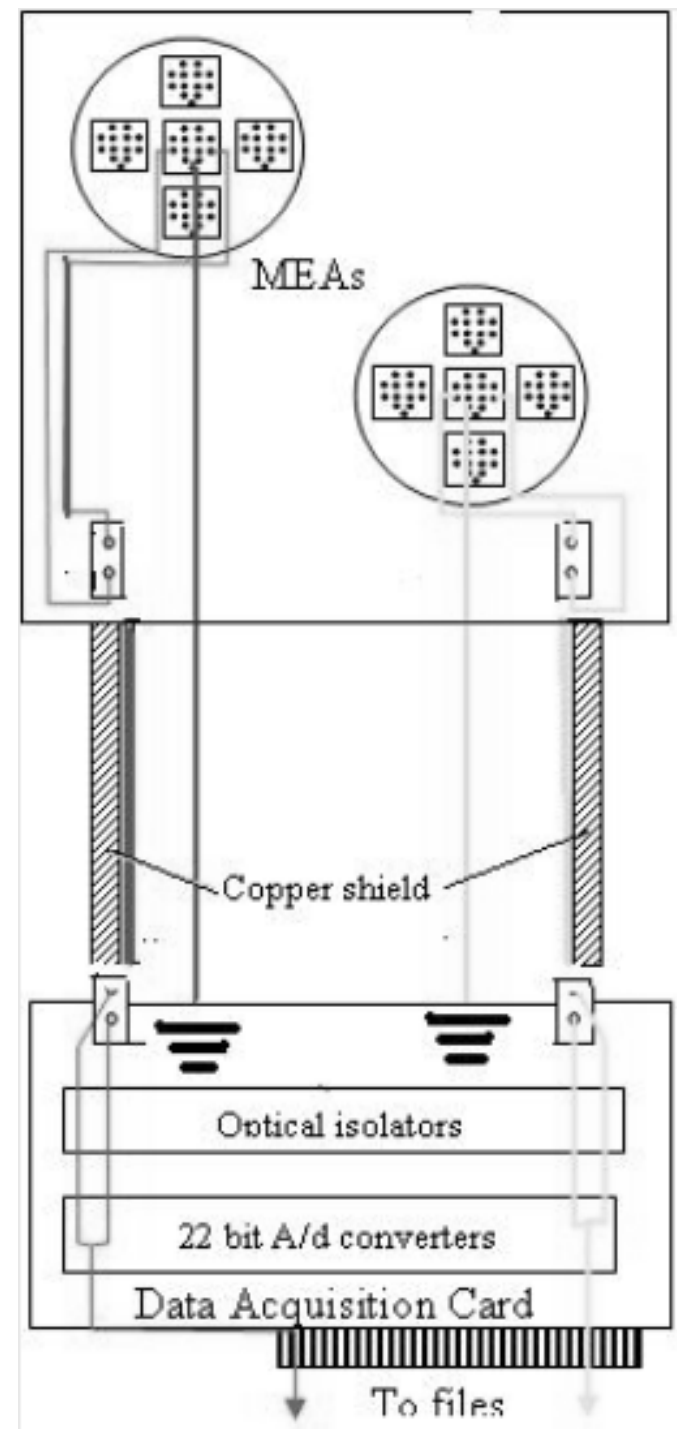

Fig. 11 MEA's and Cabeling

noise due to electromagnetic interference.

Assuming the cabling and leads are properly constructed, so that we can conclude that measured voltages actually come from cell cultures in their respective basins, the next question to be addressed is whether the stimulation of Basin-S might leak into Basin- $\mathrm{R}$ and hence the correlation in the measured signals might simply be due to the fact that both basins are responding to the same stimulus.

Information we have is that the receiver Basin- $R$ was shielded with a metal jacket surrounding the culture so that no light or other forms of EM radiation could penetrate. Figure 12 shows a photograph of the actual apparatus. The golden box is the Faraday cage. The inside showing the two basins with one wrapped in aluminum is shown in figure 13 The cables coming outside are also shielded, and the
The Microelectrode Array (MEA) and cabling used to conduct the experiment are shown in figure 11 . At the top is an MEA with four neuron growth basins consisting of a glass disk with tungsten $100 \times 100 \mu$ microelectrodes. Since it takes one month to grow neurons from a parent stem cell source four basins are available in order to conduct grow and conduct multiple experiments. For the non-locality experiment only two electrode pairs from two separate basins are wired to the two outside leads of a 40 pin flat ribbon cable. The remaining pins are grounded to act as shields. The entire cable is encased in a copper shield and is plugged into the controller box shown below the copper shield cable.

The controller interfaces the MEAs with the acquisition card and includes a high impedance amplifier. After the amplifier the signals pass through a $50 \mathrm{~Hz}$ Notch filter to eliminate power supply disturbances. Then the signals are transferred to the acquisition card after a complete isolation by means of special Texas Instruments (ISO124) electronic circuits that avoid coupling between circuits. The digitized data streams coming from the Basin-R(magenta) and Basin $\mathrm{S}$ (yellow) are then recorded in files.

Two questions arise. First, do the measured signals come from the neurons, and second, are the signals isolated.

To answer the first question signals were measured coming from basins filled with culture liquid, without cells, and compared with signals coming from basins containing cells. In the first case the pure culture liquid showed the response of a conductor while, in the second case the cell response is detected.

The second question was answered by injection of a 10volt test signal on one of the basins while measuring the voltage coming from the second basin. Cross-talk was measured to $100 \mathrm{db}$ below the input stimulation voltage and well below the 1 to 10 milli-volt range involved in the experiment. Similar measurement taken with empty basins showed no significant background

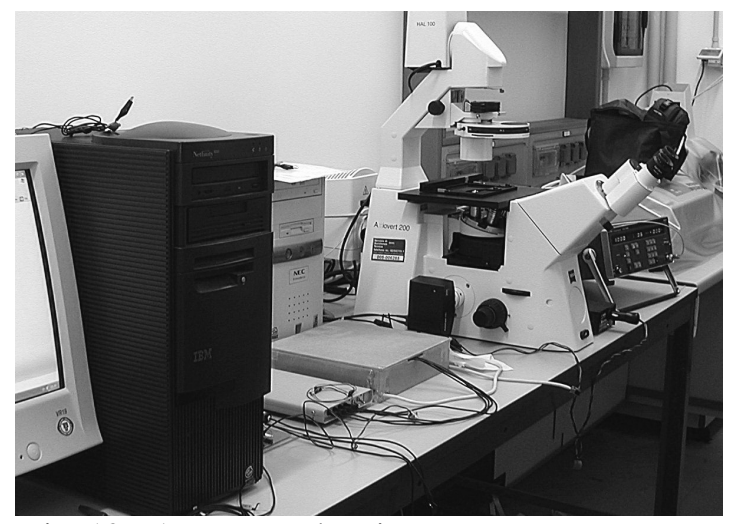

Fig. 12.- Apparatus showing EM Shielding and Faraday Cage 
small hole around the cables is also shielded. Inside on the left there is a small metallic box, the hardware controller.

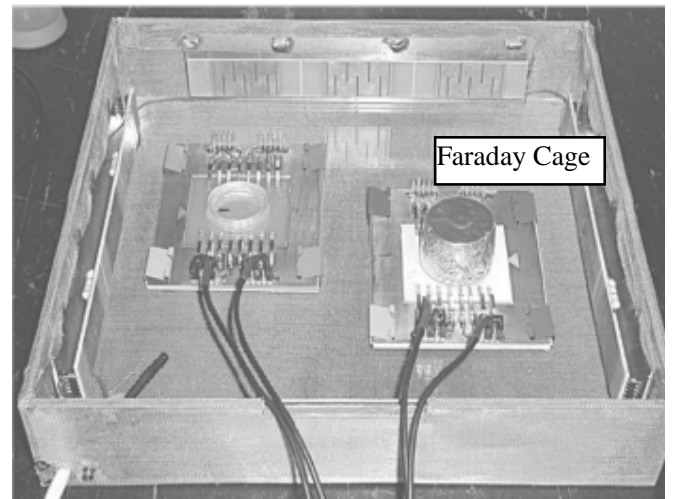

Fig. 13- Two MEA Basins

Inside Faraday Cage

\subsection{Alternative Explanation Tests}

The basic effect seen in our experiments showed that a stimulation signal produced pulses from an unshielded neuron filled basin followed by a correlated pulse from a second shielded neuron basin. To attribute such correlated signals to a quantum effect requires the elimination of all alternative possibilities. Four years of test and check experiments have been conducted with complete changes of equipment. This work is documented on line ${ }^{[29]}$ an can be accessed for a more detailed description of experiments and alternatives tested. Of primary concern is whether the observed signal correlation comes from the neuron content of the basins or some other benign component.

This concern is addressed by performing end to end tests. Such tests would maintain the identical setup as that shown in figure 11 with the exception that the neuron cell colony is replaced by a culture liquid containing fibroblasts or possibly cells of a different DNA. If the stimulation, shielding, and cabling were identical and the only difference were the material content of the MEA basins, then repeatable differences in measurement results must be attributed to the only variable in the experiment. This variable is the neuron cell culture DNA. Such end to end tests have been done. So, for example, the pairings of basin content listed in table 2 showed no measured signal correlation.

TABLE 2 End To End test with different Basin Content

$\begin{array}{lll}\text { Basin-S } & \text { Basin-R } & \text { Result } \\ \text { Culture liquid } & \text { Human Neurons } & \text { No Correlation } \\ \text { Fibroblasts } & \text { Human Neurons } & \text { No Correlation } \\ \text { Rat Neurons } & \text { Human Neurons } & \text { No Correlation }\end{array}$

Many tests were done to eliminate experimental error and false measurements. Benchmark test were conducted on the acquisition card by injecting up to $1 \mathrm{~V}$ signals on more channels with crosstalk results of $<-110 \mathrm{~dB}$. We simulated spikes in one channel and verified that they do not propagate through the other channels. Crosstalk preamplifier tests were conducted by injecting up to $80 \mathrm{mV}$ ( from 100 to $500 \mathrm{~Hz}$ ) into each channel with noise results of $<2 \mathrm{mV}$. High current sparks were injected in the preamplifier circuit with no interference shown.

Electromagnetic shielding tests were conducted by introducing an antenna in the brass Faraday cage. The antenna was connected to a spectrum analyzer, detecting frequencies from $100 \mathrm{Khz}$ to $3.5 \mathrm{GHz}$. The instrument did not detect any activity during the laser pulses. The antenna was connected to an oscilloscope to verify presence of frequencies in the range 0-100 KHz. The instrument did not detect any activity during the laser pulses.

Sound stimulation is eliminated because spikes appear only when stimulated in the experiments not in response to other noise sources of equal or greater intensity as those happening during the experiment.

We next considered the possibility that the phenomenon could be due to an electromagnetic field coming from the laser supply circuit that was too weak to be detectible with our measure instruments. To test this hypothesis we constructed a suitable circuit consisting of a selective sensor able to collect extremely weak induced electromagnetic fields that might reach the MEAs. We used a large squared ceramic capacitor $(100 \mathrm{nF})$ and connected it to a sensitive amplifier. The amplified signal was sent to the acquisition card and analyzed. The test showed a peak in the MEA receiver channel simultaneous to the laser activation. Because the same peak was present after substituting the laser with a dummy load in order 
to simulate the current absorption equivalent to the one generated by the laser, we concluded that stimulation from the laser supply circuit was the cause of the neuron response.

We believe that neurons may receive and amplify an electromagnetic spike through the air whose value, before reaching the Faraday shielding, is under the sensitivity of our instrumentation $(2 \mathrm{mV})$. The exact value under a double Faraday cage could not be measured but is estimated to be several orders of magnitude less. It is possible that the neurons are the active receiving element because the MEA basins are connected to ground, and their shape is not suitable to act as antenna and because the spikes observed in the neural basin are never present in the other control basins.

A large body of literature exists to explain, by means of super-radiance, stochastic resonance, and other theories of how the neural activity can be amplified and processed inside the cellular and extracellular system. The neural reactivity may be due to the presence of microtubules in their cellular structure. In fact microtubules are structurally and dimensionally similar to carbon nanotubes, whose quantum properties are well known. Moreover, the tubular structure of carbon nanotubes makes them natural cavity antennas; their peculiar configuration can act as an array of antennas and amplify the signal.

Hence we must conclude this section with the statement that the evidence we have been able to see suggests electronic isolation has been achieved and correlated signals observed were from isolated neuron basins, but that does not constitute completely compelling proof that quantum communication has been observed. The possibility also exists that neurons are extremely sensitive to low levels of radiation below the levels of our current instrument to detect. However observed pulse delays would speak against such as yet untested possibilities. In the future, we hope to extend the shielding by employing high Mu-metal shielding and extend our experiments to search for evidence of other quantum effects in neurons. Regardless of the final outcome of these experiments, we believe the MEA approach is an excellent method for investigating the possibility of quantum effects in biological systems and encourage other laboratories to duplicate our experiments.

\section{CONCLUSION}

We reviewed experimental procedures that might be applied in order to experimentally verify the possibility of quantum communication in biological systems and presented extensive experimental work performed to explore this possibility. The current results indicate that neurons either exhibit effects attributable to quantum phenomena or are extremely sensitive to electromagnetic radiation below levels we were able to measure. Since it is impossible to conduct experiments which prove a quantum effect by eliminating all other imaginable possibilities we strongly urge other laboratories to investigate these effects and offer our assistance to any verification efforts undertaken.

Even if verification experiments duplicate the effect it will be difficult to accept the possibility of quantum effects in a warm environment until a theoretical basis for such phenomena can be built. Toard this end we have explored an alternative interpretation of quantum theory that includes the observer not simply as a small disturbance but as an equal member of a universe in which each part executes selfmeasurement activities. In order to maintain the individual identity we actually observe in cognitive beings such parts would need to be isolated so that measurement interactions are small perturbations. This suggests the possibility that the cognitive components of biological systems are sufficiently isolated to perform quantum computations. If true, such components can serve both as guides and resources for the

construction of quantum computers. Whether or not such explanations prove valid we believe the observed response of neurons is significant and urge further experimentation to be conducted.

\section{REFERENCES}

[1] Penrose, Roger (1991), The Emperor's new Mind, Penguin Books

[2] Hameroff, S. 1998 Quantum Computing in Brain microtubules, Philosophical Transactions Royal Society London (A) 356:1869-1896 (1998) see URL for related publications http://www.quantumconsciousness.org/publications.html

[3] Stapp, H. P. (1993), Mind, Matter, and Quantum Mechanics. Springer-Verlag: Berlin

[4] Baer, Wolfgang (2006) "Amazing Light-Visions for Discovery”, Journal of Consciousness Studies, 13,No.1-2, 2006, pp.177-183 
[5] Manousakis,Efstratios (2007) Quantum theory, consciousness and temporal perception: Binocular Rivalry, Cornell University Library, http://arxiv.org/abs/0709.4516

[6] Pinker, Steven (1997), How the Mind Works, W. W. Norton \& Company Co. , p. 146

[7] Khrennikov,Andrei (2006) Brain as quantum-like computer, Cornell University Library, http://arxiv.org/PS_cache/physics/pdf/0702/0702250v2.pdf Note: extensive reference listings in the introduction

[8] Chalmers, D.J. (1997) "Facing up to the problem of consciousness", Journal of Consciousness Studies 4:3-46. Reprinted in (j. Shear,ed) Explaining Consciousness: The Hard Problem, MIT Press,. URL http://www.geocities.com/computerresearchassociated/consciousness.htm

[9] Bieberich, Erhard "Probing quantum coherence in a biological system by means of DNA amplification", Biosystems, Volume 57, Issue 2, July 2000, Pages 109-124

[10] vonNeumann,J. (1932) Mathematische Grundlagen der Quantum Mechanik, Springer Verlag, Berlin, 1932

[11] Wheeler, J. A and Zurek W. H. , (1983) Quantum Theory and Measurement, Princeton University Press. For example see "Relative State Formulation of Quantum Mechanics" by H. Everett p 315

[12] Stapp, H. P. (1979), "Whiteheadian Approach to Quantum Theory and the Generalized Bell's Theorem", Foundations of Physics 9, pp 1-25

NOTE: Personal discussions with $\mathrm{H}$. Stapp suggests that the process nomenclature does not exactly correspond to his ideas. They do however correspond to his usage of them in lectures I have hard.

[13] James, William (1890), The Principles of Psychology, Vol. I and II, Dover 1955 (see Chapter X The Consciousness of Self)

[14] Whitehead, Alfred North, (1978) "Process and Reality an Essay in Cosmology", Corrected Edition by D. R. Griffin and D. W. Sherburne, The Free Press N.Y. ISBN 0-02-934580-4

[15] Atmanspacher, H. and Hans Prines (2006) "Pauli's Ideas on Mind Matter in the Context of Contemporary Science", Journal of Consciousness Studies Vol 13, No 3, pp 34

[16] Sherburne, W. Donald (1981), A Key to Whitehead's Process and Reality, University of Chicago Press, ISBN 0-226-75293-3, Keeton, State University of New York Press, ISBN 0-7914-5913-6

[17] Nobo, Jorge L. (2004), "Whitehead and Quantum Experience", Physics and Whitehead: Quantum Process and Experience, edited by Timothy E. Eastman and Hank

[18] Clayton, Philip (2004), "Introduction to Process Thought", op. sid. Physics and Whitehead, p. 6

[19] Whitaker, Andrew (1996) Einstein, Bohr and the Quantum Dilemma,

See Chapter 8 Peierls and the Knowledge Interpretation of Quantum Theory p301 Cambridge University Press QC174.12 .W48 1996

[20] Kafatos, Menas (1989)Bell's Theorem, Quantum Theory and Conceptions of the Universe, Edited by Menas Kafatos, Kluwer Academic Publishers, p. 163

[21] Cahill, R.T. (2006) "A New Light-Speed Anisotropy Experiment: Absolute Motion and Gravitational Waves Detected", Progress in Physics, Vol 4, Oct 2006 p73-92 for a review of the evidence for an inertial space attached to fixed star backgrounds.

[22] Mensky, Michael (2006) "Reality in quantum mechanics, Extended Everett Concept, and consciousness", http://arxiv.org/abs/physics/0608309 Note: a good introduction to the extensive writings of M. Mensky on the Universe self measurement concept

[23] Baer, Wolfgang (2007) "The Physical Condition for Consciousness", Journal of Consciousness Studies, 14, No. 8, 2007, pp. 93-104

[24] Manousakis,Efstratios (2007) Quantum theory, consciousness and temporal perception: Binocular Rivalry, Cornell University Library, http://arxiv.org/abs/0709.4516

[25] R. Pizzi(2004) A. Fantasia, F. Gelain, D. Rossetti and A. Vescovi, NON-LOCAL CORRELATIONS BETWEEN SEPARATED NEURAL NETWORKS, Proc. SPIE Conference on Quantum Information and Computation, Orlando (Florida), 12-16 Aprile 2004.

[26] R. Pizzi,(2004) A. Fantasia, F. Gelain, D. Rossetti and A. Vescovi, NON LOCALITY IN BIOLOGICAL SYSTEMS ? AN EXPERIMENTAL APPROACH, Proc. Coherence 2004, 12-13 febbraio 2004, Roma, anche in: CIFA News Jan-June, 16-18, 2004

[27] R. Pizzi,(2007) G. Cino, F. Gelain, D. Rossetti and A. Vescovi, LEARNING IN HUMAN NEURAL NETWORKS ON MICROELECTRODE ARRAYS, Biosystems Journal, Volume 88, Issues 1-2, March 2007, Pages 1-15, Elsevier ed. 
[28] Jozsa, Richard (1998) "Quantum Information and its Properties", Article in Introduction to Quantum Computation and Information, Editors Hoi-Kwong Lo, Sandu Popescu, Tim Spiller, World Scientific 1998 ISBN 981023399X, see page 60

[29] www.dti.unimi.it/pizzi/SPIE09.html please down load the powerpoint slide documenting the complete set of experiments

[30] See: http://www.dti.unimi.it/ pizzi/pubbl1.html for additional publications. 